\title{
Mechanism of action of intradiscal chymopapain in the treatment of sciatica: a clinical, biochemical, and radiological study
}

\author{
J R JENNER, ${ }^{1}$ DAVID J BUTTLE, ${ }^{3}$ AND A K DIXON ${ }^{2}$
}

From the Departments of ${ }^{1}$ Rheumatology and ${ }^{2}$ Radiology, Addenbrooke's Hospital, Hills Road, Cambridge; and the ${ }^{3}$ Biochemistry Department, Strangeways Research Laboratories, Worts Causeway, Cambridge

SUMmARY Seventeen patients with intractable sciatica due to prolapse of a lumbar disc, treated by intradiscal injection of chymopapain (chemonucleolysis) were studied. Analysis of serial 24 hour urine collections showed a significant increase in urinary glycosaminoglycan after chemonucleolysis. This was not detected in four patients undergoing routine discography. Enzymic analysis of urinary glycosaminoglycan after chemonucleolysis suggested that the increase in levels was largely due to an increase in the amounts of chondroitin sulphate present, probably resulting from proteoglycan breakdown in the intervertebral disc. Eight of the patients treated by chemonucleolysis underwent serial computed tomography $(C T)$. One month after the injection the only change seen was a loss of definition of the disc prolapse, which could be interpreted as a loss of turgidity in the disc as a result of proteoglycan breakdown by chymopapain. By six months the CT of those patients whose symptoms had improved showed that the degree of disc prolapse was usually less marked and the disc margin more clearly defined, suggesting that by this stage anatomical remodelling had occurred.

Chemonucleolysis has been widely adopted as a treatment for intractable sciatica. Its efficacy has been established in double blind studies, ${ }^{12}$ and chemonucleolysis is now being recommended as the last step in the conservative treatment of sciatica before surgery. ${ }^{3}$

The mechanism by which pain relief is obtained is not clearly understood. A widely held theory is that the enzyme relieves sciatica by degrading the proteoglycan content of the intervertebral disc, causing loss of glycosaminoglycan and water, resulting in 'shrinkage' of the disc and a reduction of pressure on the nerve root. ${ }^{4}$

Chymopapain is capable of degrading proteoglycan in vitro, ${ }^{5}$ and two studies in vivo have shown a rise in urinary excretion of proteoglycan breakdown products after chemonucleolysis. ${ }^{67}$ Anatomical changes, including narrowing of the disc space and reduction in size of disc protrusion on serial CT studies, have also been reported anecdotally in successfully treated patients.

Accepted for publication 24 September 1985.

Correspondence to Dr J R Jenner, Addenbrooke's Hospital, Hills Road. Cambridge CB2 2QQ.
It has been suggested that the success of chymopapain in clinical trials may be due to spontaneous remission of symptoms, ${ }^{9}$ the act of injection into the disc itself, psychogenic factors, ${ }^{10}$ or the action of a separate agent in the formulation of the commercially available enzyme. ${ }^{11}$ The last of these possibilities is not consistent with the results of a recent trial. $^{12}$

Alternative mechanisms that have been proposed are reduction of inflammation which occurs at the interface between disc prolapse and the nerve $\operatorname{root}^{13}$ and the destruction of a chemical mediator of pain. ${ }^{14}$

In order to clarify the mechanism by which chemonucleolysis relieves sciatica the relationship between clinical results, urinary excretion of proteoglycan breakdown products, and anatomical change shown by CT has been studied in patients undergoing chemonucleolysis.

\section{Patients, materials, and methods}

PATIENTS

Seventeen patients undergoing chemonucleolysis were studied. All patients were monitored clinically, 
and 24 hour urine samples were obtained pre- and postinjection. Eight of these patients underwent serial CT.

All 17 patients, whose details are shown in Table 1 , complained of unremitting symptoms and signs typical of lumbar disc prolapse. They had undergone a period of conservative treatment, including traction, an epidural injection of local anaesthetic and corticosteroid, and a minimum of two weeks' strict bed rest, usually as inpatients. Duration of symptoms ranged from three to 24 months. Six patients had a neurological deficit causing motor weakness or reflex loss, or both, consistent with a single nerve root lesion. All patients had impairment of straight leg raising on the side of the sciatica.

Anatomical confirmation of the disc prolapse was provided initially by a lumbar radiculogram. Patients were excluded if there was radiological evidence of an extruded fragment of disc. Other excluding factors were features of multiradicular palsy, spinal canal stenosis, previous spinal surgery, inconsistencies between the clinical and radiological findings, or other problems of assessment.

INJECTION TECHNIQUE

The injection was performed using the technique described by McCulloch. ${ }^{15}$ All patients were given a premedication including $100 \mathrm{mg}$ intramuscular hydrocortisone. A discogram was performed under local anaesthetic on the single disc which was clinically and radiologically implicated. The disc was then injected with 1-2 $\mathrm{ml}$ (2000-4000 units) of chymopapain (Discase, Travenol) under a brief general anaesthetic. No adverse reactions related to the procedure were observed.

\section{CLINICAL ASSESSMENT}

The patients were assessed clinically at one, three, and six months after treatment. Pain was monitored on a five point pain score-'worse', 'unimproved', 'slightly improved', 'greatly improved', or 'symptom free'. The straight leg raise was also measured with a goniometer. Patients whose symptoms were unim-

Table 1 Patients' details

\begin{tabular}{|c|c|}
\hline Age (years) & $15-72$ (mean 38) \\
\hline \multicolumn{2}{|l|}{ Sex } \\
\hline Male & 9 \\
\hline Female & 8 \\
\hline Duration of symptoms (months) & 3-24 (mean 14) \\
\hline Neurological signs L5 & 3 \\
\hline$S 1$ & 3 \\
\hline \multirow{2}{*}{ Site of injection } & 12 \\
\hline & 5 \\
\hline
\end{tabular}

proved or worse at three months were recommended for surgical removal of the disc. Clinical success was judged as those patients who were free of symptoms or greatly improved at three months. The remainder were regarded as failures.

COLLECTION OF URINE SAMPLES

Twenty four hour urine collections into sterile containers were made on all patients. The collections from patients undergoing chemonucleolysis were supervised by nursing staff in the Rehabilitation Unit, Addenbrooke's Hospital, Cambridge. Collections were made for the 24 hours before the injections and for at least 48 hours after the injections. In 10 patients 24 hour collections were continued for four to five days.

Twenty four hour urine collections were also obtained from four patients undergoing discograms as an investigative procedure. These collections, made at the subjects' homes, represented injection controls. In all cases, as soon as possible after the completion of a 24 hour urine collection, the volume was measured and a $50 \mathrm{ml}$ sample taken and stored at $-20^{\circ} \mathrm{C}$.

MATERIALS FOR URINARY ANALYSIS

1,9-Dimethyl methylene blue (DMB) was obtained from Serva Feinbiochemica, Heidelberg, FRG. Chondroitin ABC lyase, chondroitin AC lyase, keratanase, chondroitin sulphate type A from whale cartilage, and keratan sulphate were from Miles Laboratories, Slough, England. Cetylpyridinium chloride was obtained from BDH Chemicals Ltd, Poole, England. Papain (type III) was from Sigma Chemical Co. Ltd, Poole, England. All other chemicals were of analytical reagent grade.

DETERMINATION OF GLYCOSAMINOGLYCAN CONTENT OF URINE

The method of Farndale et al was used. ${ }^{16}$ The urine sample was thawed and $0.25 \mathrm{ml}$ was mixed with 2.5 $\mathrm{ml}$ of a $16 \mu \mathrm{g} / \mathrm{ml}$ solution of DMB in $0.1 \mathrm{M}$ sodium formate buffer ( $\mathrm{pH} \mathrm{3.5)}$. The absorbance at $535 \mathrm{~nm}$ was determined immediately after mixing, and the value was compared with a standard curve for $0-100 \mu \mathrm{g} / \mathrm{ml}$ of whale chondroitin sulphate. The absorbance at $535 \mathrm{~nm}$ of a control containing $0.25 \mathrm{ml}$ of water in $2.5 \mathrm{ml}$ of DMB solution was subtracted from all other values. Internal standards were included when measuring each urine sample to check for interference.

PARTIAL PURIFICATION OF GLYCOSAMINOGLYCANS FROM URINE After thawing, the urine sample was centrifuged for 10 minutes to remove material that had precipitated 
during freezing and thawing. Precipitation with cetylpyridinium chloride was employed to obtain a fraction enriched in glycosaminoglycans. ${ }^{17}$ The precipitate was washed with ethanol, dissolved in $1 \mathrm{ml}$ of $0.02 \mathrm{M}$ sodium phosphate buffer $(\mathrm{pH} 6.8$ ) containing $1 \mathrm{mM}$ disodium ethylenediaminetetraacetate (EDTA), $2 \mathrm{mM}$ dithiothreitol, and $2 \mathrm{mg}$ papain, and incubated at $60^{\circ} \mathrm{C}$ for $60 \mathrm{~min}$. Iodoacetic acid was then added to a final concentration of 10 $\mathrm{mmol} / \mathrm{l}$, and the volume was made up to $5 \mathrm{ml}$ by the addition of $0.05 \mathrm{M}$ TRIS. $\mathrm{HCl}(\mathrm{pH} 8 \cdot 0)$.

Partial purification with cetylpyridinium chloride was chosen for the digestion studies as this allowed concentration of material from day 0 and discogram urine, for which the levels of glycosaminoglycans were too low for accurate analysis.

\section{DIGESTION OF URINARY}

G L Y COSAMINOG L Y C ANS

Digestions of the partially purified, papain digested material (above) were performed on $0.5 \mathrm{ml}$ of the sample for $10 \mathrm{~min}$ at $37^{\circ} \mathrm{C}$. Incubation mixtures contained 0.05 units of chondroitin $\mathrm{ABC}$ lyase, 0.05 units of chondroitin AC lyase, or $0 \cdot 1$ units of keratanase, and approximately $10 \mu \mathrm{g}$ of glycosaminoglycan. Digestion was monitored by loss of reaction with $\mathrm{DMB}$, and digestions of known amounts of whale chondroitin sulphate or keratan sulphate were used to optimise digestion conditions.

\section{COMPUTED TOMOGRAPHY (CT)}

The CT studies were performed on a Siemens Somaton 2 third generation machine, for which a standard routine for lumbar spine studies has been developed. ${ }^{18}$ This routine is similar to that used in other centres. ${ }^{19}$ Cuts, $4 \mathrm{~mm}$ thick, were used and the gantry was tilted to approximate the angle of each disc space in turn. Each patient was studied on three occasions. A full CT study of the lumbar spine (L3 to S1) was performed immediately before chemonucleolysis and limited (one level) cuts of the treated disc space were obtained one month and approximately six months after the procedure.

The following measurements were performed. The anteroposterior (AP) extent of the disc protrusion was estimated on each occasion. A subjective assessment of the definition of the posterior peripheral margin of the disc material was also made. An attempt was made to measure the CT attenuation of the disc material in Hounsfield units, ${ }^{20}$ but such a measurement was not found to be very reproducible on account of the problems caused by the partial volume effect.

Finally, the cross sectional area of the thecal sac was assessed by analysis of the computer stored regions of interest ${ }^{18}$; this gave some measure of the degree of thecal distortion.

\section{Results}

C LIN I C A L

At three months chemonucleolysis was successful in 10 out of 17 patients. All 10 reported their pain to be 'greatly improved', and the straight leg raising on the affected side rose to within $10^{\circ}$ of the unaffected side. The remainder, who felt that their pain was 'worse' or only 'slightly improved', underwent surgical removal of the disc, having failed to respond to treatment after three months.

The operative findings of the seven failures are summarised in Table 2. All patients were found to have a disc protrusion confined by the annulus at the level injected. One was described as having a 'very firm' disc protrusion. This was a patient who had had sciatica for two years, and it was assumed that the enzyme was unable to affect such a degenerate disc. In three patients the nerve root was found to be tethered to the underlying disc protrusion by adhesions, and this was thought to be contributing to the patients' lasting symptoms. One of these three was also found to have a previously unsuspected extruded disc fragment at the disc below the one injected. There was no obvious cause found at operation to explain the failure of the enzyme injection in two patients, and it is worth noting that one of these showed no increase in excretion of urinary glycosaminoglycan after the enzyme injection.

URINARY LEVELS OF MATERIAL REACTIVE WITH DMB AFTER CHEMONUCLEOLYSIS OR DIS COGRA M

The amount of glycosaminoglycan in the urine was measured by reaction with DMB. As seen in Fig. 1 the dose-response for the absorbance at $535 \mathrm{~nm}$ of whale chondroitin sulphate reacting with DMB was linear in water and urine, with no significant interference in urine. On average, the amount of reaction with DMB increased after chemonucleolysis, reaching a maximum two days after injection (Fig. 2). The level then fell and approached preinjection values by day 5 postinjection. The levels

Table 2 Operative findings-seven patients

\begin{tabular}{ll}
\hline Disc protrusion confined by annulus & 7 \\
Very firm disc protrusion & 1 \\
Tethering of nerve root & 3 \\
Extruded fragment at uninjected level & 1 \\
\hline
\end{tabular}




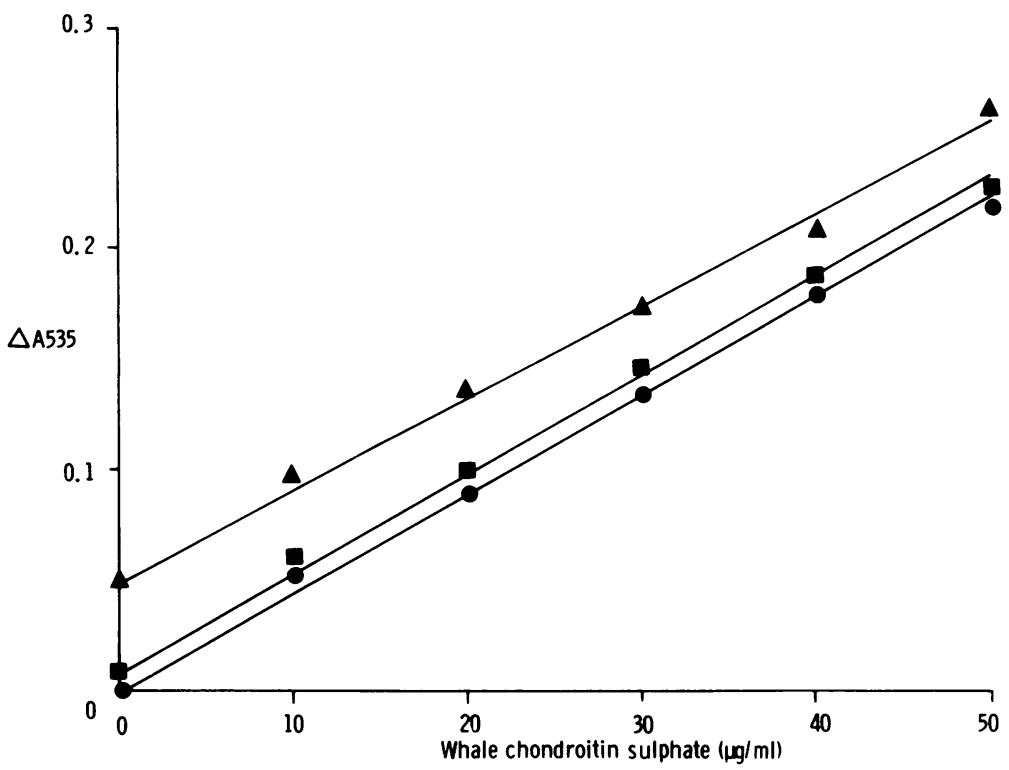

Fig. 1 Dose response for absorbance at $535 \mathrm{~nm}$ of whale chondroitin sulphate in: water (O); control urine (1) postchemonucleolysis urine $(\mathbf{\Delta}-\mathbf{\Delta})$ reacted with $D M B$.

for individual cases varied considerably. Thirteen patients showed a measurable increase in urinary material reactive with DMB. The time of maximum excretion varied between day 1 and day 5 after injection. No correlation was found between clinical improvement and urinary levels of material reactive with DMB (Fig. 3).

Four patients showed no increase in the urinary material reactive with DMB. Two were clinical failures and two were clinical successes. One of the successfully treated patients had urine collected for only 48 hours, and it is possible that a rise in urinary material reactive with DMB would have occurred after this period, as was shown for other patients who had longer collections. The other patient had the urine collections discontinued after three days because of a urinary tract infection. This infection probably interfered with the urinary levels of material reactive with DMB. ${ }^{21}$

Twenty four hour samples were also taken from patients undergoing discograms. None of these patients showed any significant increase in urinary material reactive with $\mathrm{DMB}$ after injection of contrast medium (Fig. 2), and the levels did not exceed the normal range of 8-15 $\mathrm{mg}$, expressed as chondroitin sulphate. ${ }^{22}$

\section{IDENTITY OF URINARY}

\section{G L Y COSA M INOG L Y CAN S}

Specific glycosaminoglycanases were used to identify components precipitated by cetylpyridinium chloride. Recovery of material reactive with DMB after precipitation was about $80 \%$.

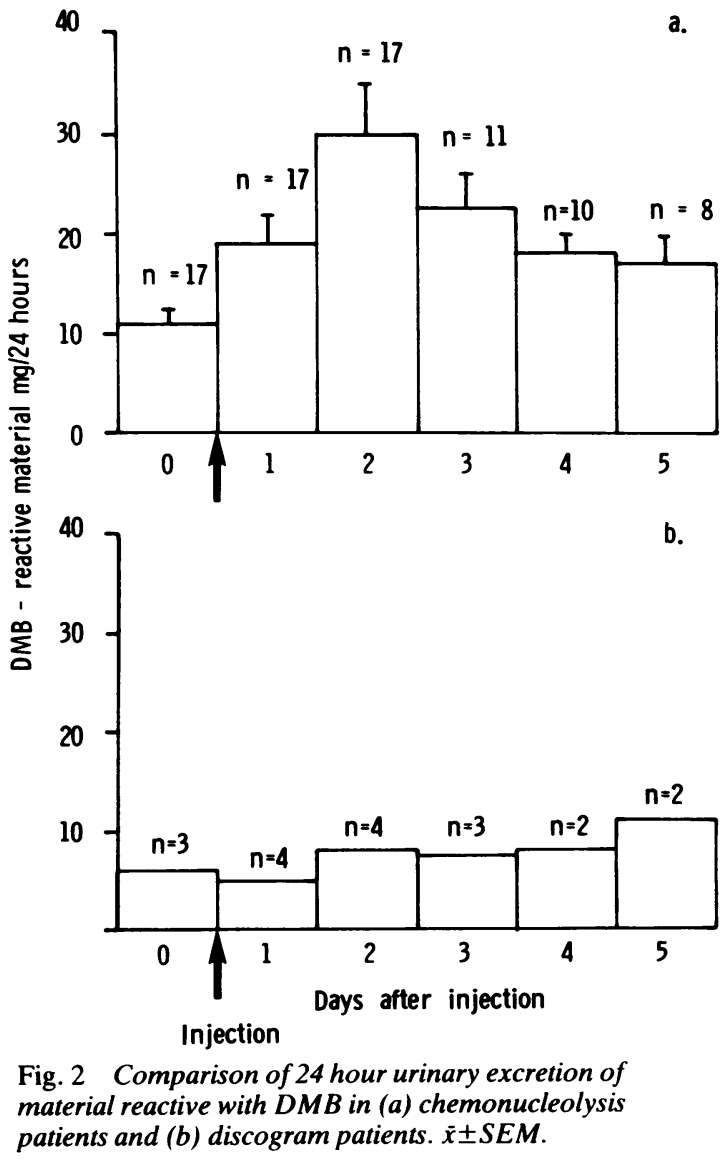




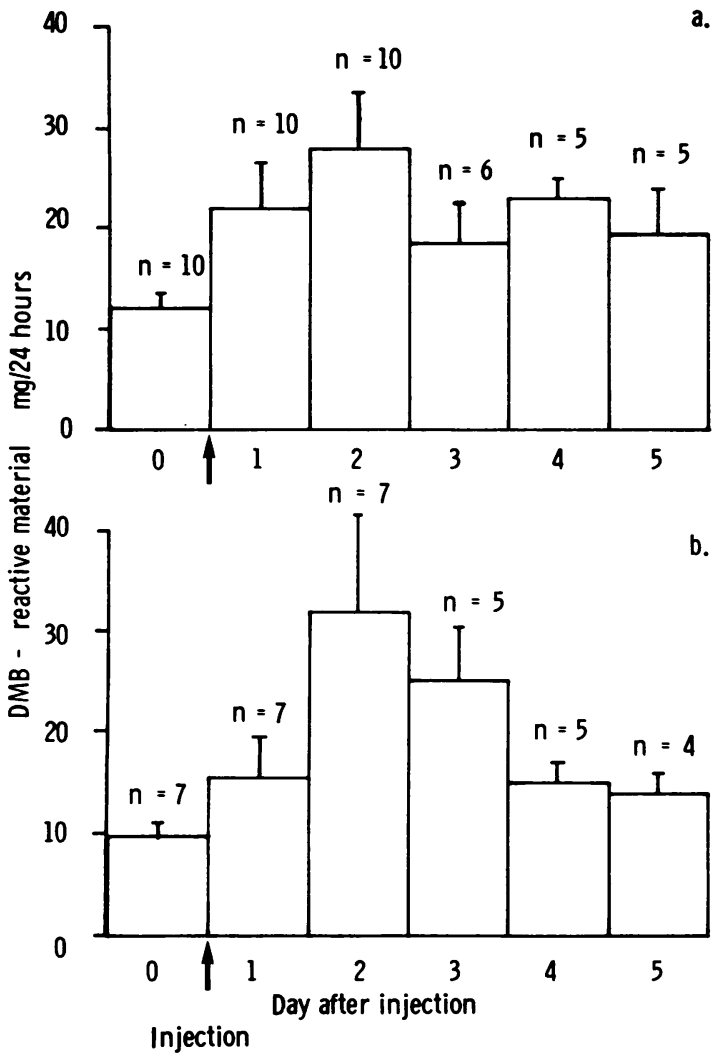

Fig. 3 Comparison of 24 hour urinary excretion of material reactive with $D M B$ in (a) clinically successful patients and (b) clinical failures. $\bar{x} \pm S E M$.

Papain digestion of the precipitates obtained by cetylpyridinium chloride treatment resulted in the removal of about $40 \%$ of the reactivity with DMB in material from preinjection urine, and about $30 \%$ in material from postinjection urine. The material digested by papain was probably composed of acidic glycoproteins, which are coprecipitated with glycosaminoglycans from urine, and may react to some extent with DMB. ${ }^{23} 24$

The specificity of the glycosaminoglycanases was confirmed as the chondroitinases failed to digest keratan sulphate under the conditions used, and keratanase failed to digest whale chondroitin sulphate.

Table 3 compares the results obtained with enzyme digestion of control (day 0) urine samples and the peak excretion day (usually day 2) of seven patients who showed a marked rise in material reactive with DMB after chemonucleolysis. It can be seen that about $50 \%$ of the material resistant to papain, reactive with DMB, and obtained by precipitation of preinjection urine with cetylpyridinium chloride was resistant to hydrolysis by any of the enzymes tested. This figure fell to about $25 \%$ from postinjection urine. The nature of this material was not investigated. Most of the remaining material was susceptible to both chondroitin $\mathrm{ABC}$ lyase and chondroitin AC lyase and was, therefore, either chondroitin-4-sulphate or chondroitin-6-sulphate. The percentage of material reactive with DMB and susceptible to the chondroitinases increased significantly after chemonucleolysis, showing that the proportion of chondroitin sulphate in the DMB reactive material and the total level of this material in the urine (Fig. 2) increased after injection of chymopapain.

The level of digestion achieved with keratanase also increased after chemonucleolysis (Table 3 ). The similarity in the amount of digestion achieved with chondroitin AC lyase and chondroitin ABC lyase suggests that very little of the material was dermatan sulphate.

\section{COMPUTED TOMOGRAPHY}

Of the eight patients undergoing serial CT, five were clinical successes and three were failures. The three failures had surgery before the third CT study was performed at six months.

The CT studies at one month showed little change in the maximum AP diameter of the disc protrusion, varying by less than $1 \mathrm{~mm}$ from the preinjection scan. The thecal sac cross sectional area also showed little change, with only one patient having an increase greater than $10 \mathrm{~mm}^{2}$. The most consistent change was the observation that in all but two patients the interface between disc material and the thecal sac was less sharply defined at one month after chemonucleolysis than before. In the remaining two patients no appreciable change in definition at one month was observed. This observation would

Table 3 Percentage of material resistant to papain and reactive with $D M B$ which was susceptible to cleavage by specific glycosaminoglycanases from urine of chemonucleolysis patients

\begin{tabular}{llc}
\hline & Before injection & After injection \\
\hline Chondroitin ABC lyase & $49 \cdot 1 \pm 2 \cdot 7^{*}$ & $70 \cdot 1 \pm 1 \cdot 4^{*}$ \\
Chondroitin AC lyase & $50 \cdot 2 \pm 1 \cdot 8^{* *}$ & $72 \cdot 0 \pm 1 \cdot 3^{* *}$ \\
Keratanase & $1 \cdot 7 \pm 1 \cdot 1^{* * *}$ & $5 \cdot 7 \pm 1 \cdot 0^{* * *}$ \\
\hline
\end{tabular}

$\mathbf{n}=7$.

Values are mean \pm standard error of the mean.

Analysis was by paired $t$ test.

${ }^{*} \mathrm{p}<0.001 ;{ }^{* *} \mathrm{p}<0.001 ;{ }^{* * *} \mathrm{p}<0.05$. 
446 Jenner, Buttle, Dixon

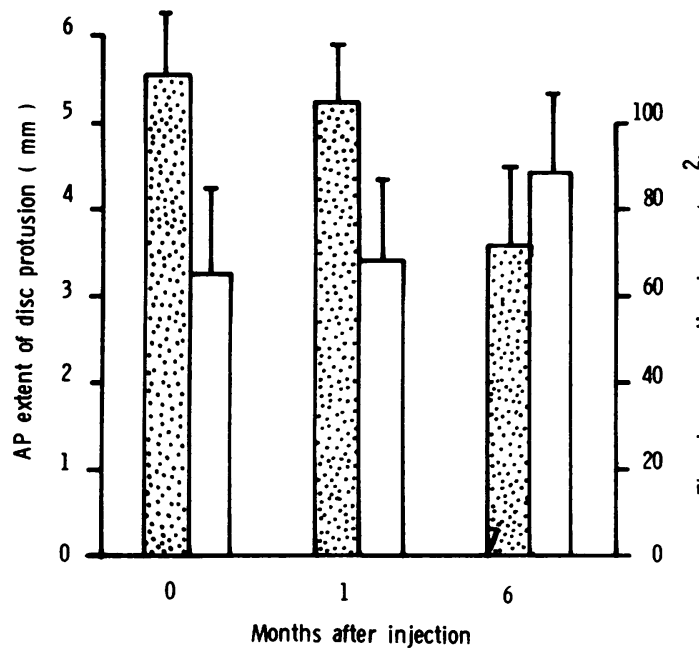

Fig. 4 The CT findings showing anteroposterior extent of disc protrusion in $\mathrm{mm}$ (dotted columns) and the available thecal cross sectional area in $\mathrm{mm}^{2}$ (white columns) preinjection and at one and six months after chemonucleolysis. $\bar{x} \pm S E M$.

be expected to vary considerably with technique but, since a very consistent radiographic routine was adopted it seems likely that such lack of definition at one month is a valid observation.

At six months three of the clinically successful patients showed a reduction in the disc protrusion by $3 \mathrm{~mm}$, the other two changing by $1 \mathrm{~mm}$ or less. The thecal cross sectional area increased in all the clinically successful patients, showing an increase of between 9 and $40 \mathrm{~mm}^{2}$ (mean $23 \mathrm{~mm}^{2}$ ), with an increase in the available thecal space of greater than $20 \%$ in four of the patients (Fig. 4). The lack of definition of the disc observed in the CT studies at one month was not a prominent feature in the five patients who underwent CT at six months, only one being judged still to have poor definition of the disc protrusion.

Fig. 5 illustrates the changes occurring in three consecutive CT studies in one of the clinically successful patients who failed to show a marked reduction in the size of the disc protrusion over a six month period. The preinjection study (Fig. 5a) shows a central disc protrusion (AP extent $7 \mathrm{~mm}$, cross sectional thecal area $43 \mathrm{~mm}^{2}$ ) with an additional focal bulge just right of centre. At one month (Fig. 5b) the posterior aspect of the protrusion was less well defined, but the AP extent of the protrusion and the thecal area remained virtually unchanged (AP extent $8 \mathrm{~mm}$, thecal area $40 \mathrm{~mm}^{2}$ ). By six months (Fig. 5c) the protrusion was more
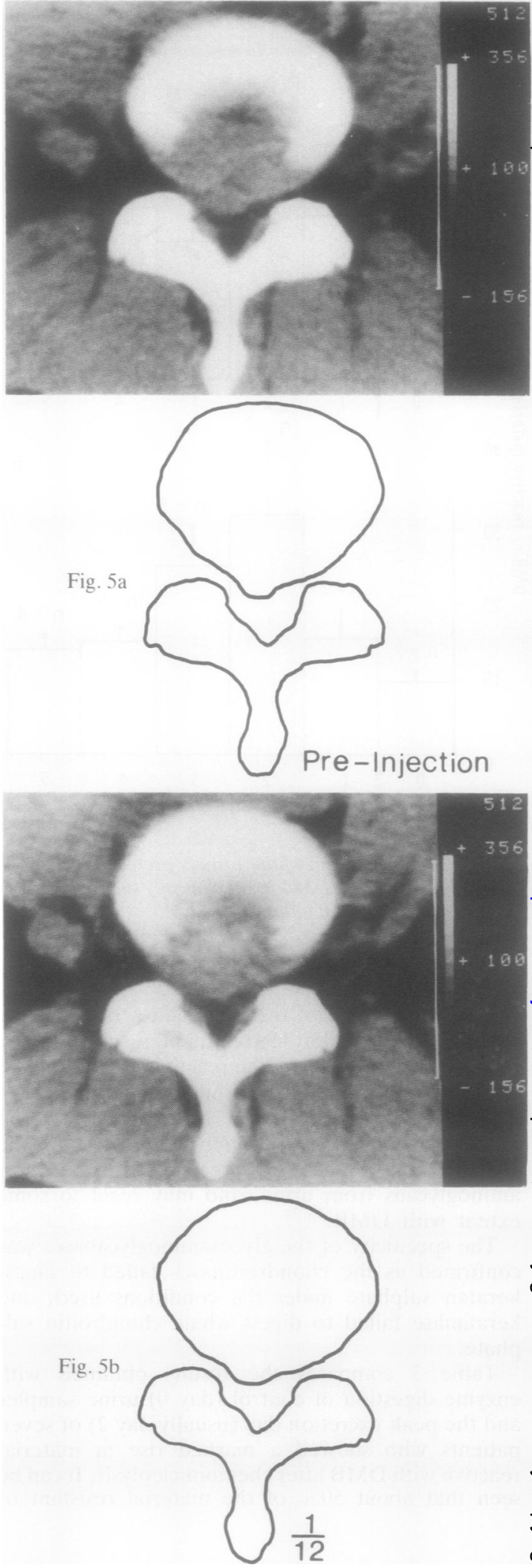


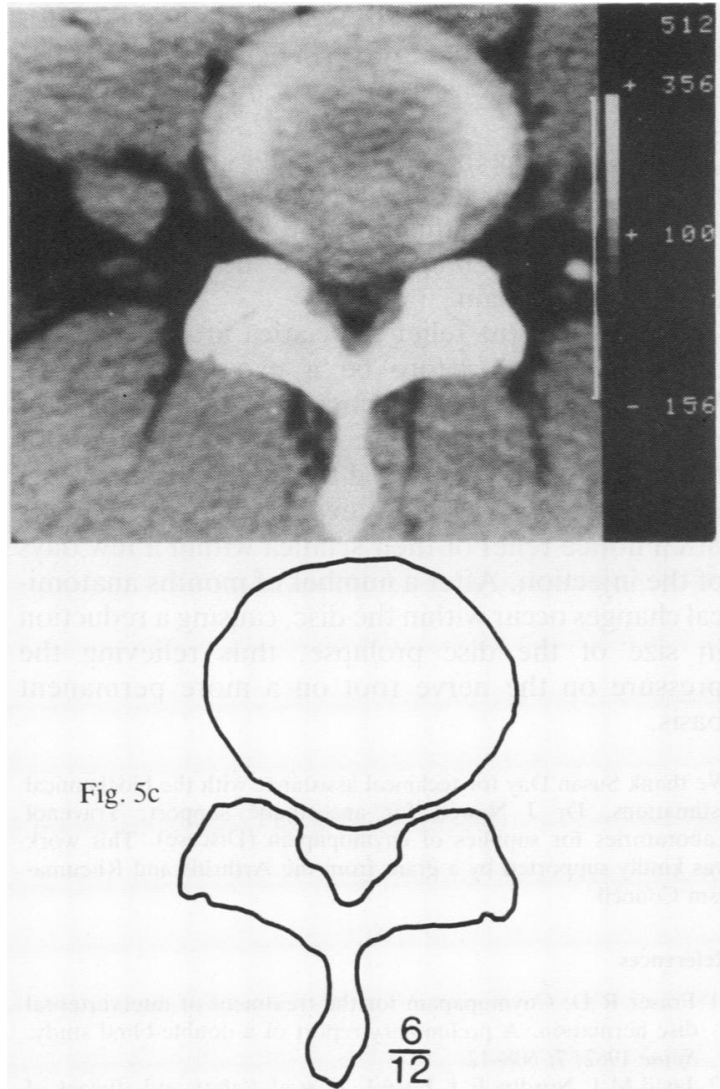

Fig. 5 Serial CT studies on patients: (a) preinjection; (b) one month after injection; and (c) six months after injection. See text for description.

generalised without the focal lesion, and although the AP extent of the protrusion remained unchanged $(7 \mathrm{~mm})$, the cross sectional area increased to $52 \mathrm{~mm}^{2}$.

\section{Discussion}

The method we have adopted for the measurement of glycosaminoglycan in urine was developed for the measurement of glycosaminoglycan release from cartilage cultures, ${ }^{16}$ and has recently been used successfully for the determination of glycosaminoglycan content of kidney basement membranes. ${ }^{25}$ The products of digestion of chondroitin-4-sulphate with testicular hyaluronidase (mainly tetrasaccharides) are fully active in the reaction with DMB, whereas the disaccharide products of digestion with the glycosaminoglycan lyases give no colour reaction. ${ }^{26}$ This method will therefore quantify sulphated glycosaminoglycans of tetrasaccharide or larger. Although we have shown that other, proteinaceous, components of urine also give a positive reaction with $\mathrm{DMB}$, the method can be successfully used in conjunction with specific polysaccharidases to provided an accurate measure of the quantity of specific glycosaminoglycans in urine.

The results of such analyses show an increase in urinary excretion of glycosaminoglycans in the majority of patients receiving chymopapain. This supports previous reports employing different methods of analysis, which also showed an increased excretion of glycosaminoglycans in the urine after chemonucleolysis. $^{67}$

A lack of correlation between clinical success and urinary levels of material reactive with DMB is not surprising as the operative findings (Table 2) suggest that clinical failure is more dependent on factors such as tethering of the nerve root than failure of enzyme to break down the proteoglycan in the disc.

The finding that the control subjects undergoing discography showed no significant rise in the urinary material reactive with $D M B$ indicates that increased levels of glycosaminoglycan after injection are not simply a response to injury caused by the injection. These results, on the contrary, strongly suggest that the increase in the urine of material reactive with DMB after chemonucleolysis is due to the degradation of the intervertebral disc proteoglycan by chymopapain.

The increases in the total amount of urinary material reactive with DMB after chemonucleolysis and in the proportion of this material which is chondroitin sulphate are consistent with the extra material being of cartilaginous origin. The appearance of some keratan sulphate in urine after chemonucleolysis is consistent with the breakdown of intervertebral disc proteoglycan, which contains substantial quantities of keratan sulphate compared with cartilage from other sources. ${ }^{27} 28$ The similarity in the proportion of the material sensitive to chondroitin $\mathrm{ABC}$ lyase and chondroitin $\mathrm{AC}$ lyase is evidence that very little dermatan sulphate was present in the urine. Human intervertebral disc contains negligible quantities of dermatan sulphate. $^{27}$

It is possible to determine the approximate amount of extra glycosaminoglycan excreted as a result of chymopapain injection at one level to be about $50 \mathrm{mg}$. This provides a minimal estimate of the amount of glycosaminoglycan mobilised by chemonucleolysis because the proportion of glycosaminoglycan excreted, as opposed to metabolised, is not known. Kaplan and Meyer reported that most chondroitin sulphate injected intravenously was not recovered in the urine. ${ }^{29}$ Revell and Muir, however, recovered undegraded chondroitin sulphate from 
urine when the samples were collected aseptically. ${ }^{21}$ The value of $50 \mathrm{mg}$ excreted can be compared with the total amount of glycosaminoglycan in human nucleus pulposus, about $170 \mathrm{mg}$, estimated using a figure of $3.0 \mathrm{~g}$ for the weight of human lumbar nucleus pulposus ${ }^{30}$ and the values for degree of hydration and percentage of dry weight due to glycosaminoglycan quoted by Saklatvala. ${ }^{31}$

The delay between injection of chymopapain and the detection of increased levels of urinary material reactive with DMB (often 48 hours or more) is probably related to the time needed for the diffusion of degraded proteoglycan from the avascular nucleus pulposus to the nearest capillary bed in the cartilaginous endplates. The delay is unlikely to be due to a build up of proteoglycan breakdown products in the blood as Bowness and Parkinson failed to find increased amounts of glycosaminoglycan in serum after chemonucleolysis. Kaplan and Meyer reported the delay between intravenous injection of dermatan sulphate and the appearance in the urine to be minutes rather than days, ${ }^{29}$ although a recent study showed that the passage of glycosaminoglycans through the glomerulus was restricted. ${ }^{24}$ Residual chymopapain may remain for a considerable time in the disc, so that proteoglycan degradation could be continuing, leading to later appearance of some glycosaminoglycan in the urine..$^{32} 33$

The CT findings in this small series are revealing. In the first month after the injection there was virtually no change in the objective measurements of the disc protrusion in any of the patients, whether or not there was clinical improvement. The most consistent change at one month was the loss of definition between the thecal sac and the disc material. This change might be due to a loss of turgidity within the disc causing the annulus to become less rigid and thus less sharply defined, lending further support to the theory that chemonucleolysis reduces pressure on the nerve root by reducing the hydrostatic pressure within the prolapse, rather than removing the prolapse itself. That the prolapse still remains one month after the injection is perhaps not surprising in view of the observed lack of action of this enzyme on collagen.

Within six months of the injection the definition of the disc margin on CT had almost returned to normal in those patients whose clinical condition improved. More obvious anatomical changes, however, had also occurred, with reduction in the size of the disc prolapse and a reciprocal increase in the available cross sectional area in the majority of the patients. At this stage the turgidity of the disc may have returned, so that the sciatica is now relieved by the anatomical 'shrinkage' of the disc away from the nerve root. The delay of between one and six months might represent the time taken for $\frac{\nabla}{\varphi}$. the collagenous structure of the disc to reorganise after the loss of a large proportion of its proteoglycan content.

It is interesting that major changes in anatomy are $\frac{}{O}$ not essential for the relief of sciatica and subtle $\frac{\overline{\bar{c}}}{\overline{\frac{1}{2}}}$ changes in the outline of the disc prolapse, as $\frac{\widehat{\phi}}{\circ}$ illustrated in Fig. 5, appear to be sufficient to result on in the relief of pain.

The permanent relief of sciatica after chemonucleolysis may therefore be a two stage process. $\overrightarrow{\vec{\omega}}$ Within days of the injection there is release of $\stackrel{\omega}{S}$ proteoglycan from the disc, which results in loss of turgidity within the disc and the relief of pressure on the nerve root, perhaps explaining why patients of often notice relief of their sciatica within a few days of the injection. After a number of months anatomi- $\nexists$ cal changes occur within the disc, causing a reduction $ᄋ$ in size of the disc prolapse, thus relieving the pressure on the nerve root on a more permanent $c_{\subset}$ basis.

We thank Susan Day for technical assistance with the biochemical $\vec{\emptyset}$ estimations, Dr J Newell for anaesthetic support. Travenol o Laboratories for supplies of chymopapain (Discase). This work was kindly supported by a grant from the Arthritis and Rheumatism Council.

\section{References}

1 Fraser R D. Chymopapain for the treatment of intervertebral disc herniation. A preliminary report of a double-blind study. Spine 1982; 7: 608-12.

2 Javid M J, Nordby E J, Ford L T, et al. Safety and efficacy of chymopapain (Chymodiactin) in herniated nucleus pulposus with sciatica. Results of a randomised double-blind study. JAMA 1983; 249: 2489-94.

3 Crawshaw C. Frazer A M. Merriman W F, Mulholland R C, Webb $\mathbf{J}$ K. A comparison of surgery and chemonucleolysis in the treatment of sciatica. Spine 1984; 9: 195-8.

4 Smith L, Brown J E. Treatment of lumbar intervertebral disc lesions by direct injection of chymopapain. J Bone Joint Surg O 1967; 49B: 502-19.

5 Buttle D J, Tudor J, Barrett A J. Effect of $x$-ray contrast media $\bigcirc$ on the action of chymopapain on the intervertebral disc: an in vitro study of cartilage degradation. $B r J$ Radiol 1984; 57: $\frac{D}{2}$ 475-7.

6 Bowness J M. Parkinson D. Increased glycosaminoglycan excretion after chymopapain injection of intervertebral discs. o Clin Biochem 1983; 16: 200-1.

7 Stern I J, Cosmas F, Smith L. Urinary polyuronide excretion in man after enzymic dissolution of the chondromucoprotein of $\omega$ the intervertebral disc or surgical stress. Clin Chim Acta 1968; 무 21: 181-90.

8 McCulloch J A, Macnab 1. Sciatica and chymopapain. Balti- $\frac{\varrho}{\frac{C}{(1)}}$ more: Williams and Wilkins, 1983; 191-5.

9 Sussman B J. Inadequacies and hazards of chymopapain $\stackrel{\mathscr{C}}{+}$ injections as treatment for intervertebral disc disease. $J$ Neuro- $\square$ surg 1975; 42: 389-96.

10 Martins A N, Ramirez A, Johnston J, Schwetzchenau P R. Double-blind evaluation of chemonucleolysis for herniated lumbar discs. Late results. J Neurosurg 1978; 49: 816-27.

11 Brown M D, Daroff R B. The double-blind study comparing $\sigma$ Discase to placebo. An editorial comment. Spine 1977; 2: 233-6. 
12 Morris J M, Stromberg L. Double-blind study of Discase (chymopapain) versus CE1 (chymopapain activator) in the treatment of herniated nucleus pulposus in the lumbar spine. Proceedings the International Society for the Study of the Lumbar Spine 1983.

13 Branemark P, Ekholm R, Lundskog J, Hirsch C. Tissue response to chymopapain in different concentrations. Animal investigations on microvascular effects. Clin Orthop Rel Res 1969; 67: 52-67.

14 Macnab I, McCulloch J A , Weiner D S, Hugo E P, Galway R D, Dall D. Chemonucleolysis. Can J Surg 1971; 14: 280-9.

15 McCulloch J A. Chemonucleolysis. J Bone Joint Surg 1977; 59B: $45-52$.

16 Farndale R M, Sayers C A, Barrett A J. A direct spectrophotometric microassay for sulphated glycosaminoglycans in cartilage cultures. Connect Tissue Res 1982; 9: 247-9.

17 Barrett A J, Sledge C B, Dingle J T. Effect of cortisol on the synthesis of chondroitin sulphate by embryonic cartilage. Nature 1966; 211: 83-4.

18 Dixon A K. Body CT, a handbook. Edinburgh: Churchill Livingstone, 1983: 113-6.

19 Chafetz N T, Genant H K. Mani J R. Computed tomography of the spine: technical considerations. In: Moss A A, Gamsu G. Genant H K, eds. Computed tomography of the body. Philadelphia: Saunders, 1983: 427-36.

20 Teplick J G, Haskin M E. CT and lumbar disc herniation. Radiol Clin North Am 1983; 21: 259-88.

21 Revell $\mathrm{P} A$, Muir $\mathrm{H}$. The excretion and degradation of chondroitin 4-sulphate administered to guinea pigs as free chondroitin sulphate and as proteoglycan. Biochem J 1972; 130: 597-606.

22 Diferrante N, Rich C. The determination of acid aminopolysaccharide in urine. J Lab Clin Med 1956; 48: 491-4.

23 Satake S, Ototani N, Isemura M, Yosizawa Z. Enzymatic determination of urinary glycosaminoglycans from orthopaedic patients. Tohoku J Exp Med 1983; 140: 89-96.

24 Shum D K Y, Baylis C, Scott J E. A micropuncture and renal clearance study in the rat of the urinary excretion of heparin, chondroitin sulphate and metabolic breakdown products of connective tissue proteoglycans. Clin Sci 1984; 67: 205-12.

25 Reubsaet F A G, Langeveld J P M, Veerkamp J H. Glycosaminoglycan content of glomerular and tubular basement membranes of various mammalian species. Biochim Biophys Acta 1985; 838: 144-50.

26 Farndale R W, Buttle D J, Barrett A J. Improved quantitation and discrimination of sulphated glycosaminoglycans by use of dimethylmethylene blue. Biochim Biophys Acta (in press).

27 Gurr E, Schubert R, Delbruck A. A sensitive method for the analysis of the glycosaminoglycan distribution pattern, applied to 9 intervertebral discs of one human spine. J Clin Chem Clin Biochem 1982; 20: 723-9.

28 Stevens R L, Ewins R J F, Revell P A, Muir H. Proteoglycans of the intervertebral disc. Homology of structure with laryngeal proteoglycans. Biochem J 1979; 179: 561-72.

29 Kaplan D, Meyer K. The fate of injected mucopolysaccharides. J Clin Invest 1962; 41: 743-9.

30 Hendry N G C. The hydration of the nucleus pulposus and its relation to intervertebral disc derangement. J Bone Joint Surg 1958; 40B: 132-44.

31 Saklatvala J. The non-collagenous matrix of cartilage. Structure, physiology and changes with age and disease. In: Panayi, G S, ed. Scientific basis of rheumatology. Edinburgh: Churchill Livingstone, 1982: 159-78.

32 Kapsalis A A, Stern I J, Bornstein I. The fate of chymopapain injected for therapy of intervertebral disc disease. J Lab Clin Med 1974; 83: 532-40.

33 Bradford D S, Cooper K M, Oegema T R. Chymopapain, chemonucleolysis and nucleus pulposus regeneration. $J$ Bone Joint Surg 1983; 65A: 1220-31. 